\title{
Social media supporting disaster response: Evaluation of a lightweight collaborative tool
}

\author{
Beth Veinott, Ph.D. \\ Klein Associates Division of ARA \\ bveinott@ara.com
}

\author{
Donald Cox, M.S. \\ Klein Associates Division of ARA \\ dcox@ara.com\
}

\author{
Shane Mueller, PhD. \\ Klein Associates Division of ARA \\ smueller@ara.com
}

\begin{abstract}
Motivation: Disaster response efforts rely heavily on ad hoc, cross organization teams with multiple stakeholders, who must collaboratively make sense of the situation, and are often working at a distance. These properties make it difficult to design collaborative tools in support of decision making in these environments. Research Approach: In this paper we use virtual ethnographic techniques as a way to bridge the gap between important contextual and socio-technical conditions and early tool assessment. For an example case of some socio-technical issues, we discuss Twitter use during the San Diego, California wildfires in October 2007. Findings: We found that Twitter supported some aspects of distributed collaboration well, but not others. Originality: This research provided an early examination of the collaboration this form of connectivity supports which can be applied in several domains.
\end{abstract}

\section{Keywords}

Collaboration, Social Media, Design, Virtual Ethnography, Disaster Response

\section{INTRODUCTION}

Collaborative systems have unique challenges relative to single-user systems. They can change the group processes (Dourish, 2001; Veinott \& Irwin, 1993), have organizational and cultural impacts by violating norms and roles (Orlikowski, 1992; Olson \& Olson, 2003) and fail to achieve critical mass (Grudin, 1992). Consequently, evaluating collaborative systems requires more contextual approaches than single user systems. In this study we use a virtual ethnographic approach for identifying patterns of collaboration that support decision making in disaster response operations. We analyze the use of a Web 2.0 tool, Twitter, a social networking and micro-blogging service in which users can send and read short posts. We examine how twitter supports the socio-technical requirements of collaborative information sharing during crisis response.

\section{DESIGN REQUIRMENTS}

In responding to disasters, people need lightweight, low bandwidth tools to provide information about current status of people, roads, electricity, traffic, and damages for effective decision making. Response teams and civilians need enough information about the situation to be useful, but not too much information to adversely impact their decisions as they may not have the time, bandwidth, or screen space to wade through mounds of information. Finally, people need to develop an appropriate picture of the nature of the situation. Social media tools support emergent distributed collaboration and decision making of civilians and first responders in unique ways (Paylen \& Liu, 2007).

\section{RESEARCH DOMAIN AND APPROACH}

Twitter is a social networking and micro-blogging service in which users can send, receive, and read short posts that can provide just in time information for crisis management. Twitter, broadly launched in October 2006, is a platform independent, lightweight subscriber information source. It allows people to post messages or micro-blogs up to 140 characters making it an effective communication tool for mobile devices and adaptive to emergent situations. People can subscribe to be "followers" of an individual or of a topic (e.g., disaster) allowing people to quickly share relevant information with their family, friends, and others.

During the October 2007 Southern California wildfires in the United States, over 6000 firefighters, U.S. Armed Forces and U.S. National Guard responded to the San Diego wildfires and facilitated the evacuation of over 250,000 residents. Many people on the ground posted micro-blogs of disaster related information (fire locations, status of individual streets, and capacity of rescue centers) on Twitter to help people in Southern California with information to make real time decisions. In addition to needing to collaborate and coordinate in joint operations, civilians and the local public radio station, KPBS, were posting information to a variety of sources. 


\section{RESULTS}

Twitter supports emergent collaboration among many users and could potentially support command and control in joint disaster response operations in several ways. Twitter crosses platforms as it can be accessed via the web or cell phone making it effective for information sharing in joint operations (first responders, military and civilian). Therefore, as in radio broadcasts, many people can benefit from the Twitter posts of one person providing an effective lightweight mechanism for disseminating information. However, unlike a radio broadcast, Twitter does not rely on a single user or single location making it more resilient to power outages. Finally Twitter can provide close to real time information to support situation awareness and decision making. Example posts ('tweets') on Twitter during the wildfires were:

\section{Areas in and around Wildcat Canyon Road south of the Barona Reservation are now open for repopulation. \\ The only wildfire burning in Los Angeles County was about 80 percent contained tonight, according to the U.S. Forest Service}

There are also a few challenges for Twitter as a lightweight collaborative tool in terms of situation awareness, information overload, and coordinating information. First, because Twitter is limited to 140 characters, it provides little context making it difficult to visualize the information that was needed in responding to the wildfires. For example, in a Tweet above, if one is not familiar with the area around Wildcat Canyon Road then he will not know how to use this information. Recognizing this need, Google started working around the clock to update their maps with up to date fire location information to provide this kind of visualization. The 140 character limit is useful to keep the posts short and on point reducing information overload, but the vast number of them make it difficult to coordinate information. A second issue that arose was being able to find the wildfire relevant information. Another emergent practice was adding a tag, (e.g., \#sandiegofire) to each post so people could find the wildfire relevant posts more easily. These two challenges would need to be addressed to make it more effective for joint operations in disaster response.

\section{CONCLUSION}

People are highly adaptive when it comes to using information technologies. Many collaborative tools have short lifecycles. Consequently, the design and use of such systems naturally co-evolves. We learn a lot about the emergent properties of collaboration by studying communication on these systems. For these tools to be effectively used and adopted, the context, the social structures, and the collaborative situation need to be evaluated together. We were able to do that because of the virtual nature of Twitter and our results provide a couple collaboration patterns that should be useful for early evaluation of other lightweight collaborative technologies.

\section{ACKNOWLEDGEMENTS}

We thank the U.S. Army's Advanced Decision Architectures Collaborative Technology Alliance for sponsoring this research under the Cooperative Agreement DAAD19-01-2-0009. The views and conclusions contained in this document are those of the authors and should not be interpreted as representing the official policies, either expressed or implied, of the U.S. Army Research Laboratory or the U.S. Government.

\section{REFERENCES}

Dourish, P. (2001). Where the action is: Foundations of embodied interaction. MIT Press: Cambridge.

Grudin, J. (1999) Interactive systems: Bridging the gap between developers and users. In R. Baecker, J. Grudin, W. Buxton, and S. Greenberg (Eds) Human Computer Interaction: Toward the year 2000.(2 ${ }^{\text {nd }}$ Edition).pp. 293-304.

Orlikowski, W. (1992). Learning from notes: Organizational issues in groupware implementation. In Proceedings of the 1992 ACM conference on Computer-supported work. pp. 362-369. Toronto, Ontario, Canada.

Olson, J. S. and Olson, G. M. (2003). Cultural Surprise in Remote Software Teams. ACM Queue, 9, 52-59.

Veinott, E. S. and Irwin, C. M. (1993). Analysis of communication in the standard versus automated aircraft. In Proceedings of the Seventh International Symposium on Aviation Psychology (pp. 584-588) Columbus: Ohio State University Press.

Paylen, L. and Liu, S. B. (2007). Citizen communication in Crisis: Anticipating a Future of ICT-Supported Public Participation. In Proceedings of the 1992 ACM CHI conference on Computer Human Interaction (pp. 727-736), San Jose, California, USA. 